\title{
Quo vadis DRG?
}

\section{Jürg Unger-Köppel}

Dr. med., Mitglied des FMH-Zentralvorstandes, Departementsverantwortlicher Stationäre Versorgung und Tarife

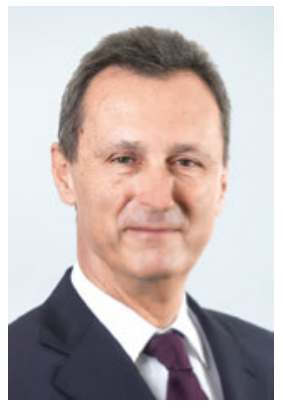

Tarife im Sozialversicherungsbereich sind Kompromisse - sie werden von den Partnern im Gesundheitswesen entwickelt, die unterschiedliche Interessen vertreten. Sie können zudem nicht vom Anbieter allein festgelegt oder nur verhandelt werden, sondern die Tarifstrukturen sind vielmehr stets datenbasiert $\mathrm{zu}$ berechnen.

Seit der Einführung der DRG im Jahr 2012 wurde das System jedes Jahr durch die SwissDRG AG weiterentwickelt. Ab 2018 ist bereits die Version 7.0 gültig, sofern der Bundesrat der aktualisierten Version zustimmt. Wir sind als FMH in die partnerschaftliche Entwicklung der Tarife eng mit eingebunden. So nehmen wir Ärztinnen und Ärzte Einfluss auf die Entwicklung der Tarife. Wir zeigen von der FMH jedes Jahr genau, wo die Veränderungen zur letzten Tarifversion erfolgten und werten diese Entwicklungsarbeiten aus ärztlicher Sicht. Die aktuelle Stellungnahme zur SwissDRG Version 7.0 finden Sie in dieser Ausgabe der Schweizerischen Ärztezeitung auf Seite 935.

Eine übergeordnete Sicht ist bei der zukünftigen Ausgestaltung des Fallpauschalensystems SwissDRG unbedingt zu priorisieren.

In der diesjährigen Würdigung der aktuellsten Version weisen wir erneut darauf hin, dass die Entwicklung aus unserer Sicht noch zu wenig von einer übergeordneten Sicht geprägt ist. Die jährlichen Neuerungen sind geprägt von Reaktion: Jedes Jahr können die Fachgesellschaften und Dachverbände, Kantone, Krankenversicherer und Spitäler Anträge an SwissDRG stellen, wo der Tarif noch Unwuchten aufweist und korrigiert werden sollte. Auf Grund der Datenauswertung wird dann entschieden, ob der Antrag zu einer Korrektur der Tarifstruktur führt oder nicht. Wünschenswert wäre aus Sicht der FMH zusätzlich auch Aktion in Form von übergeordneten Antworten auf Fragen wie: Wie lassen sich Hochdefizitfälle langfristig reduzieren? Wie kann man Fehlanreize durch Hochgewinnfälle vermeiden? Wie geht es weiter an der Schnittstelle zur Ambulanz?
Kann man das System vereinfachen und damit den administrativen Aufwand für die Leistungserbringer senken? Gibt es Entwicklungspotential über die DRG hinaus für einzelne Gebiete der Somatik? Wie sollen die drei stationären Tarifsysteme (SwissDRG, TARPSY, ST Reha) Kinder und Jugendliche sinnvoll abbilden?

Eine qualitativ hochstehende Datenbasis schafft erst die Voraussetzung für eine überzeugende Tarifstruktur.

Die FMH ist zusammen mit $\mathrm{H}+$, Versicherern und der Gesundheitsdirektorenkonferenz Besitzerin der SwissDRG AG. Im Verwaltungsrat und allen Arbeitsgruppen vertreten wir die Interessen der Ärzteschaft. Weil die meisten Fragen auf der Basis von Datenauswertungen beantwortet werden, müssen die Daten den Anforderungen genügen. Das folgende Beispiel zeigt, dass die Daten auch der Intuition widersprechen können: Die meisten denken, dass ein Fall, der notfallmässig aufgenommen wird, höhere Kosten verursacht, als ein regulärer Eintritt. Die bisherigen Daten ergeben aber, dass dies über alle Fälle genommen weder bei den DRG noch bei TARPSY der Fall ist. Die Resultate und damit die Tarifstrukturen können nur so gut sein, wie die Daten, auf denen sie beruhen. Initiativen «unserer» SwissDRG AG, zusammen mit den Spitälern deren Datenqualität zu verbessern, sind daher sehr zu begrüssen.

Der Teufel liegt bekanntlich im Detail, denn selbst wenn die Ärzteschaft an der Front alle Patientenbehandlungen gut dokumentiert, bedeutet das noch lange nicht, dass die Daten über die verschiedenen Schnittstellen hinweg richtig bei der SwissDRG AG ankommen. Es ist deshalb immer wieder von zentraler Bedeutung, dass alle an diesen Datenlieferungsprozessen Beteiligten die Plausibilität der Daten überprüfen. So schaffen wir gemeinsam eine qualitativ hochstehende Datenbasis, um Antworten auf Fragen und Entwicklungen zu geben. Dank dieser kann die FMH die Meinung der Ärztinnen und Ärzte in diesem Entwicklungsprozess nachhaltig vertreten. 